\title{
Law of the iterated logarithm for random walks on nilpotent groups
}

\author{
LUCIA CARAMELLINO and VALENTINA DI VINCENZO \\ Dipartimento di Matematica, Università Roma Tre, Largo San Leonardo Murialdo 1, I-00146 \\ Roma, Italy.E-mail: lucia@mat.uniroma3.it
}

We prove a law of the iterated logarithm for products of independent, identically distributed random variables taking values on simply connected, graded, nilpotent Lie groups as a consequence of similar results, of functional type, for stochastic processes which are solutions of ordinary differential equations with random coefficients.

Keywords: iterated logarithm; large and moderate deviations; nilpotent Lie groups; random walks

\section{Introduction}

Let $\left\{Z^{j}\right\}_{j}$ be a sequence of independent and identically distributed (i.i.d.) random variables taking values on a simply connected, graded, nilpotent Lie group $G$ and consider the product

$$
T^{n}=Z^{1} \circ \cdots \circ Z^{n}, \quad n \geqslant 1,
$$

where $\circ$ is the product operation on $G$. In this paper we show a law of the iterated logarithm for the random walk $\left\{T^{n}\right\}_{n}$ by providing the set of its limit points whenever it is suitably normalized by means of the sequence $\{\sqrt{n \ln \ln n}\}_{n}$. The normalization we shall use is the usual one which has already been used to prove several asymptotic properties for $\left\{T^{n}\right\}_{n}$ as $n \rightarrow \infty$. Specifically, it is constructed through the Lie algebra with which the simply connected, graded, nilpotent group $G$ can be identified, as follows.

Let $\mathfrak{G}$ stand for the Lie algebra of $G$. $\mathfrak{G}$ is said to be graded if it admits the decomposition

$$
\mathfrak{G}=V_{1} \oplus \cdots \oplus V_{l},
$$

where $V_{i}, i=1, \ldots, l$, are vector subspaces of $\mathfrak{b}$ such that $\left[V_{i}, V_{j}\right] \subseteq V_{i+j}$, where $V_{i+j}=\{0\}$ if $i+j>l$. Thus, any $g \in \mathbb{G}$ can be uniquely decomposed as

$$
g=\sum_{i=1}^{l} P_{i} g,
$$

where $P_{i}$ is the projection on $V_{i}$. For any $\gamma \in \mathbb{R}$, we can define the linear map

$$
D_{\gamma} g=\sum_{i=1}^{l} \gamma^{i} P_{i} g
$$


which is an endomorphism of the Lie algebra $\mathbb{G}$.

The group $G$ is said to be graded if $\mathscr{G}$ is. By assuming that $G$ is simply connected, it follows that it can be identified with through the Campbell-Hausdorff formula, so that the transformation $D_{\gamma}$ acts on $G$ too.

Consider now a random variable $Z$ taking values on $G$. We say that there exists $\mathrm{E}\left[|Z|^{\beta}\right]$ if such a moment exists whenever $Z$ is considered as a random variable on the Euclidean space $\mathbb{G}$. We say that $Z$ is centred on $G$ if its projection $P_{1} Z$ on $V_{1}$ is centred as a random variable on the Euclidean space $V_{1}$. Lastly, we say that the Laplace transform of $Z$ exists (eventually in a neighbourhood of the origin) if the same transform exists for the random variable $Z$ taking values on the Euclidean space $\mathbb{G}$.

Returning to the random walk $\left\{T^{n}\right\}_{n}$, let us normalize it by means of the map defined in (1). The asymptotic behaviour of $\left\{D_{\gamma_{n}} T^{n}\right\}_{n}$ as $n \rightarrow \infty$ differs according to how fast the sequence $\left\{\gamma_{n}\right\}_{n} \subset \mathbb{R}_{+}$goes to 0 as $n \rightarrow \infty$. For example, when $\gamma_{n}=1 / n$, if $Z^{j}$ is centred on $G$ then

$$
D_{1 / n} T^{n} \rightarrow 0 \text { almost surely }
$$

as $n \rightarrow \infty-$ that is, a strong law of large numbers holds; see, for example, Theorem 3.9 in Neuenschwander 1996, p. 101. Assuming, moreover, that $Z^{j}$ has a finite Laplace transform, then the laws induced by $\left\{D_{1 / n} T^{n}\right\}_{n}$ on $G$ satisfy a large-deviation principle at speed $n-$ that is, there exists a rate function $I_{L}$ strictly depending on the law of the $Z^{j}$, such that

$$
-\inf _{g \in A} I_{L}(g) \leqslant \liminf _{n \rightarrow \infty} \frac{1}{n} \ln P\left(D_{1 / n} T^{n} \in A\right) \leqslant \liminf _{n \rightarrow \infty} \frac{1}{n} \ln P\left(D_{1 / n} T^{n} \in A\right) \leqslant-\inf _{g \in \bar{A}} I_{L}(g),
$$

a fact which has been proved in Baldi and Caramellino (1999).

If instead $\gamma_{n}=1 / \sqrt{n}$, assuming that $\mathrm{E}\left[\left|Z^{j}\right|^{2+\delta}\right]$ is finite for some $\delta>0$, it has been shown that $\left\{D_{1 / \sqrt{n}} T^{n}\right\}_{n}$ converges in law to a random variable which can be represented as the value at time 1 of the principal diffusion on $G$ - that is, a sort of Donsker invariance principle holds; see Caramellino et al. (1999) and references quoted therein.

Suppose now that $\gamma_{n}$ goes to 0 as $n \rightarrow \infty$ faster than $1 / \sqrt{n}$ but slower than $1 / n$ :

$$
\lim _{n \rightarrow \infty} \sqrt{n} \gamma_{n}=0, \quad \lim _{n \rightarrow \infty} n \gamma_{n}=+\infty
$$

In such a case, assuming that the Laplace transform of $Z^{j}$ is finite in a neighbourhood of the origin, then the sequence $\left\{D_{\gamma_{n}} T^{n}\right\}_{n}$ satisfies a moderate-deviation principle at speed $\left(\sqrt{n} \gamma_{n}\right)^{-2}$ - that is, there exists a rate function $I_{M}$ which does not depend on both the law of the $Z^{j}$ and the sequence $\left\{\gamma_{n}\right\}_{n}$ satisfying (2), such that

$$
-\inf _{g \in A} I_{M}(g) \leqslant \liminf _{n \rightarrow \infty} n \gamma_{n}^{2} \ln P\left(D_{\gamma_{n}} T^{n} \in A\right) \leqslant \liminf _{n \rightarrow \infty} n \gamma_{n}^{2} \ln P\left(D_{\gamma_{n}} T^{n} \in A\right) \leqslant-\inf _{g \in \bar{A}} I_{M}(g),
$$

a result proved in Baldi and Caramellino (1999).

In this paper, we consider a particular sequence $\left\{\gamma_{n}\right\}_{n}$ for which (2) holds: setting

$$
\gamma_{n}=\frac{1}{\sqrt{n \ln \ln n}}
$$

we study the asymptotic behaviour of $\left\{D_{\gamma_{n}} T^{n}\right\}_{n}$ by giving explicitly the set of its limit 
points, a result which can be interpreted as a law of the iterated logarithm for random walks on nilpotent Lie groups.

This statement has been already introduced in Baldi and Caramellino (1999), where a precise proof is not given. Here, we give the proof of such a result as a consequence of a functional law concerning a more general class of processes. Indeed, the idea underlying the proof is to represent $D_{1 / \sqrt{n \ln n}} T^{n}$ as the value at time 1 of the solution of a suitable ordinary differential equation (ODE) with stochastic coefficients, whose randomness is driven by a normalized polygon constructed by means of the sequence $\left\{Z^{j}\right\}_{j}$. In Section 2 we consider this more general kind of process, for which we show that a functional law of the iterated logarithm holds. We make considerable use of some moderate-deviation estimates proved in Baldi and Caramellino (1999). Section 3 is devoted to the study of random walks on nilpotent groups: we represent $\left\{D_{1 / \sqrt{n \ln \ln n}} T^{n}\right\}_{n}$ as the value at time 1 of a suitable sequence of processes whose asymptotic behaviour is analysed in Section 2, so that a law of the iterated logarithm follows. Finally, we consider the Heisenberg group, by comparing our result with that proved by Crepel and Roynette (1977).

\section{A functional result for polygons}

Let $(\Omega, \mathscr{F}, P)$ be a probability space where a sequence $\left\{Z^{j}\right\}_{j}$ of $m$-dimensional i.i.d. random variables is defined. We suppose that the $Z^{j}$ are centred and that the Laplace transform exists in a neighbourhood of the origin.

We also suppose that the covariance matrix of the $Z^{j}$ is equal to the identity, an assumption which will be relaxed later.

Let

$$
S_{t}=\sum_{k=1}^{[t]} Z^{k}+(t-[t]) Z^{[t]+1}=S_{[t]}+(t-[t])\left(S_{[t]+1}-S_{[t]}\right),
$$

with $S_{0}=0$. Since $S$ is piecewise linear, $\dot{S}_{t}=Z^{[t]+1}, t>0$, exists almost everywhere, so that one can define the process $X_{t}$ as the solution of the ODE with stochastic coefficients

$$
\begin{aligned}
\dot{X}_{t} & =\hat{b}\left(X_{t}\right)+\hat{\sigma}\left(X_{t}\right) \dot{S}_{t}, \\
X_{0} & =x,
\end{aligned}
$$

where $\hat{b}: \mathbb{R}^{d} \rightarrow \mathbb{R}^{d}$ and $\hat{\sigma}: \mathbb{R}^{d} \rightarrow \mathbb{R}^{d} \otimes \mathbb{R}^{m}$ are respectively a vector and a matrix field that must satisfy certain conditions in order to guarantee the existence of the solution $X_{t}$ for $t>0$ (we shall specify the proper conditions on $\hat{b}$ and $\hat{\sigma}$ later).

The aim of this section is to prove a law of the iterated logarithm for the process $X$ : by means of a suitable family of contractions $\left\{\Gamma_{a}\right\}_{\alpha}>0$, we shall show that the sequence $\left\{Y^{n}\right\}_{n}$, defined by

$$
Y_{t}^{n}=\Gamma_{\sqrt{n \ln \ln n}}\left(X_{n t}\right), \quad t \in[0,1],
$$

as a.s. relatively compact on the space $\mathscr{C}\left([0,1], \mathbb{R}^{d}\right)=\left\{\varphi:[0,1] \rightarrow \mathbb{R}^{d} \mid \varphi\right.$ is continuous $\}$.

We first set 


$$
S_{t}^{n}=S_{n t}=\sum_{k=1}^{[n t]} Z^{k}+(n t-[n t]) Z^{[n t]+1}, \quad t \in[0,1],
$$

as the continuous polygon having value $S_{n}=\sum_{k=1}^{n} Z^{k}$ at time $k / n$, and linear between $k / n$ and $(k+1) / n$, so that we can write

$$
\frac{\mathrm{d}}{\mathrm{d} t}\left(X_{n t}\right)=n \dot{X}_{n t}=n \tilde{b}\left(X_{n t}\right)+\tilde{\sigma}\left(X_{n t}\right) \dot{S}_{t}^{n}
$$

because $\dot{S}_{t}^{n}=n \dot{S}_{n t}$.

For a fixed $\alpha>0$, consider now the map

$$
\Gamma_{\alpha}: \mathbb{R}^{d} \rightarrow \mathbb{R}^{d}
$$

satisfying the following hypothesis, first introduced in Baldi (1986) in the framework of diffusion processes.

Assumption 2.1. For any fixed $\alpha>0, \Gamma_{\alpha}$ is a $\mathscr{C}^{1}$ map such that:

(i) $\Gamma_{\alpha}(x)=x$;

(ii) if $\alpha \geqslant \beta$, then

$$
\left|\Gamma_{\alpha}(y)-\Gamma_{\alpha}(z)\right| \leqslant\left|\Gamma_{\beta}(y)-\Gamma_{\beta}(z)\right|
$$

for any $y, z \in \mathbb{R}^{d}$;

(iii) $\Gamma_{\alpha}^{-1}=\Gamma_{\alpha^{-1}}$ and, for any compact set $C \subset \mathbb{R}^{d}$ and $\varepsilon>0$, there exists $\delta>0$ such that if $|\alpha \beta-1|<\delta$ then

$$
\sup _{y \in C}\left|\Gamma_{\alpha} \circ \Gamma_{\beta}(y)-y\right|<\varepsilon
$$

It is worth remarking that condition (i) ensures that $x$, the starting point of the process $X$ defined in (4), is a fixed point for $\Gamma_{\alpha}$, for any $\alpha>0$. Moreover, condition (ii) stands for a sort of contraction as $\alpha$ varies: for any $y, z \in \mathbb{R}^{d}$, the distance between $\Gamma_{\alpha}(y)$ and $\Gamma_{\alpha}(z)$ decreases as $\alpha$ increases. Finally, condition (iii) ensures a sort of continuity property for $\Gamma_{\alpha}$ with respect to the parameter $\alpha$ uniformly on the compact subsets of $\mathbb{R}^{d}: \Gamma_{\alpha} \circ \Gamma_{\beta}$ is not far from the identity map whenever $\alpha \cdot \beta$ is near 1 .

Setting $\alpha_{n}=\sqrt{n \ln \ln n}, n>2$, we can now define

$$
Y_{t}^{n}=\Gamma_{\alpha_{n}}\left(X_{n t}\right)
$$

We will now study the asymptotic behaviour of $\left\{Y^{n}\right\}_{n}$ as a sequence of random variables on the space of the continuous paths, by proving that $\left\{Y^{n}\right\}_{n}$ is a.s. relatively compact in $\mathscr{C}\left([0,1], \mathbb{R}^{d}\right)$ and giving explicitly the set of its limit points. We shall rely heavily on suitable moderate-deviation estimates proved in Baldi and Caramellino (1999).

By (9) and (7), we have

$$
\begin{aligned}
\dot{Y}_{t}^{n} & =b_{n}\left(Y_{t}^{n}\right)+\sigma_{n}\left(Y_{t}^{n}\right) \frac{1}{\alpha_{n}} \dot{S}_{t}^{n}, \\
Y_{0}^{n} & =x,
\end{aligned}
$$


where

$$
\begin{aligned}
& b_{n}(y)=\left.n \nabla_{z} \Gamma_{\alpha_{n}}(z) \hat{b}(z)\right|_{z=\Gamma_{\alpha_{n}}^{-1}(y)}, \\
& \sigma_{n}(y)=\left.\alpha_{n} \nabla_{z} \Gamma_{\alpha_{n}}(z) \hat{\sigma}(z)\right|_{z=\Gamma_{\alpha_{n}}^{-1}(y)},
\end{aligned}
$$

in which, as usual,

$$
\left(\nabla_{z} \Gamma_{\alpha}(z)\right)_{i j}=\frac{\partial}{\partial z_{j}}\left(\Gamma_{\alpha}(z)\right)_{i}
$$

Since $\alpha_{n} / \sqrt{n} \rightarrow+\infty$ and $\alpha_{n} / n \rightarrow 0$, representation (10) ensures that the sequence $\left\{Y^{n}\right\}_{n}$ satisfies a moderate-deviation principle subject to the following hypothesis on the coefficients $b_{n}$ and $\sigma_{n}$ defined in (11):

Assumption 2.2. (i) $b_{n}$ and $\sigma_{n}$ are locally Lipschitz continuous, uniformly in $n$.

(ii) There exists a vector field $b$ and a matrix field $\sigma$ such that

$$
\lim _{n \rightarrow \infty} b_{n}=b, \quad \lim _{n \rightarrow \infty} \sigma_{n}=\sigma
$$

uniformly on the compact subsets of $\mathbb{R}^{d}$.

(iii) $\sigma$ is differentiable and $b, \sigma, \sigma^{\prime}$ are locally Lipschitz continuous.

(iv) Denote by $\mathscr{C C}_{0}^{m}$ the set of the absolutely continuous paths from $[0,1]$ to $\mathbb{R}^{m}$, starting at 0 , and $v: \mathscr{C}\left([0,1], \mathbb{R}^{m}\right) \cap\left\{h \mid h_{0}=0\right\} \rightarrow \mathbb{R}_{+}$,

$$
v(h)= \begin{cases}\frac{1}{2} \int_{0}^{1}\left|h_{s}^{\prime}\right|^{2} \mathrm{~d} s & \text { if } h \in \mathscr{C}_{\mathscr{C}_{0}^{m}}^{m} \text { and } h^{\prime} \in L^{2}([0,1]), \\ +\infty & \text { otherwise. }\end{cases}
$$

Then, for any $h \in\{v<\infty\}$, the Cauchy problem

$$
\begin{aligned}
& \varphi_{t}^{\prime}=b\left(\varphi_{t}\right)+\sigma\left(\varphi_{t}\right) h_{t}^{\prime}, \\
& \varphi_{0}=x,
\end{aligned}
$$

has a unique solution $\varphi$ defined on the entire time interval $[0,1]$.

As has been proved in Baldi and Caramellino (1999), Theorem 4, we have:

Theorem 2.3. Under Assumption 2.2, $\left\{Y^{n}\right\}_{n}$ satisfies a moderate-deviation principle on $\mathscr{C}\left([0,1], \mathbb{R}^{d}\right)$ at speed $\alpha_{n}^{2} / n=\ln \ln n$, with rate function

$$
J(\varphi)= \begin{cases}\int_{0}^{1} \mathscr{L}\left(\varphi(t), \varphi^{\prime}(t)-b(\varphi(t))\right) \mathrm{d} t & \text { if } \varphi \in \mathscr{b}_{x}^{d}, \\ +\infty & \text { otherwise }\end{cases}
$$

where $\mathscr{L}(\xi, \cdot)$ denotes the Legendre transform of $\theta \mapsto \frac{1}{2}\left\langle\theta, \sigma(\xi) \sigma^{t}(\xi) \theta\right\rangle, \theta \in \mathbb{R}^{d}, \mathscr{C O}_{x}^{d}$ 
standing for the set of absolutely continuous paths of $\mathscr{C}\left([0,1], \mathbb{R}^{d}\right)$, starting at $x$. Symbolically,

$$
-\inf _{\varphi \in \AA} J(\varphi) \leqslant \liminf _{n \rightarrow \infty} \frac{1}{\ln \ln n} \ln P\left(Y^{n} \in A\right) \leqslant \liminf _{n \rightarrow \infty} \frac{1}{\ln \ln n} \ln P\left(Y^{n} \in A\right) \leqslant-\inf _{\varphi \in \bar{A}} J(\varphi)
$$

for any Borel set $A \subset \mathscr{C}\left([0,1], \mathbb{R}^{d}\right)$.

Moreover, the following inequality holds: for any $R, \eta, a>0$ and $h \in\{v \leqslant a\}$, there exists $\delta>0$ such that

$$
\limsup _{n \rightarrow \infty} \frac{1}{\ln \ln n} \ln P\left(\left\|\frac{1}{\alpha_{n}} S^{n}-h\right\|<\delta,\left\|Y^{n}-F(h)\right\| \geqslant \eta\right) \leqslant-R,
$$

where $F=F_{x}$ is defined by

$$
\begin{aligned}
F_{x}:\{v<\infty\} & \rightarrow \mathscr{C}\left([0,1], \mathbb{R}^{d}\right) \\
h & \mapsto F_{x}(h)_{t}=x+\int_{0}^{t} b\left(F_{x}(h)_{s}\right) \mathrm{d} s+\int_{0}^{t} \sigma\left(F_{x}(h)_{s}\right) h_{s}^{\prime} \mathrm{d} s .
\end{aligned}
$$

Remark 2.4. It is worth recalling for later use some properties to Theorem 2.3.

(i) The sequence $\left\{(1 / \sqrt{n \ln \ln n}) S^{n}\right\}_{n}$ also satisfies a moderate-deviation principle on $\mathscr{C}\left([0,1], \mathbb{R}^{m}\right)$, at speed $\ln \ln n$, with rate function $v$ defined in (12):

$$
\begin{aligned}
-\inf _{h \in A} v(h) & \leqslant \liminf _{n \rightarrow \infty} \frac{1}{\ln \ln n} \ln P\left(\frac{1}{\sqrt{n \ln \ln n}} S^{n} \in A\right) \\
& \leqslant \liminf _{n \rightarrow \infty} \frac{1}{\ln \ln n} \ln P\left(\frac{1}{\sqrt{n \ln \ln n}} S^{n} \in A\right) \leqslant-\inf _{h \in \bar{A}} v(h),
\end{aligned}
$$

for any Borel set $A \subset \mathscr{C}\left([0,1], \mathbb{R}^{m}\right)$. This is an obvious consequence of Theorem 2.3, once one chooses $d=m, \hat{b}=0$ and $\hat{\sigma}=I d$, but this is actually a well-known result in the literature, proved during the 1970s by Mogul'skii (see Mogul'skii 1976; but also Baldi and Caramellino 1999 and references quoted therein). Notice that the form of the rate function suggests that $\left\{(1 / \sqrt{n \ln \ln n}) S^{n}\right\}_{n}$ turns out to have the same asymptotic behaviour that such a sequence should have if the $Z^{j}$ were standard Gaussian random variables.

(ii) The rate functions $J$ and $v$ are lower semi-continuous functionals such that the level sets $\{\varphi: J(\varphi) \leqslant a\}$ and $\{h: v(h) \leqslant a\}$ are compact subsets of $\mathscr{C}\left([0,1], \mathbb{R}^{d}\right)$ and $\mathscr{C}\left([0,1], \mathbb{R}^{m}\right)$ respectively, for any $a \geqslant 0$. Moreover, for any $\varphi \in \mathscr{C}\left([0,1], \mathbb{R}^{d}\right)$ for which $J(\varphi)$ is finite, there exists $h \in \mathscr{C}\left([0,1], \mathbb{R}^{m}\right)$ such that

$$
\varphi=F_{x}(h) \quad \text { and } \quad J(\varphi)=v(h)=\frac{1}{2} \int_{0}^{1}\left|h^{\prime}(s)\right|^{2} \mathrm{~d} s .
$$

Indeed, it can be proved (see, for example, Baldi and Caramellino 1999, p. 803) that $J(\varphi)=\inf _{h \in F_{x}^{-1}(\{\varphi\})} v(h)$. If $a=J(\varphi)$ is finite, set $C_{a}=F_{x}^{-1}(\{\varphi\}) \cap\{h: v(h) \leqslant a+1\}$, so that $J(\varphi)=\inf _{h \in C_{a}} v(h)$. Now, $C_{a}$ is compact because the level set $\{h: v(h) \leqslant a+1\}$ is 
compact and the set $F_{x}^{-1}(\{\varphi\})$ is closed because $F_{x}$ is a continuous functional on $\{h: v(h) \leqslant a+1\}$ (see Baldi and Caramellino 1999, Appendix). Since $v$ is lower semicontinuous, the infimum of $v$ on $C_{a}$ is achieved, and the assumption above actually holds.

By using the estimates given by Theorem 2.3, we shall prove the following result, which can be interpreted as a law of the iterated logarithm for the process $X$ :

Theorem 2.5. Under Assumptions 2.1 and 2.2, the sequence $\left\{Y^{n}\right\}_{n}$ is a.s. relatively compact in the space $\mathscr{C}\left([0,1], \mathbb{R}^{d}\right)$ and the set of its limit points as $n \rightarrow \infty$ is given by

$$
K=\{\psi: J(\psi) \leqslant 1\},
$$

with $J$ as defined in (13).

Remark 2.6. Before giving the proof of this result, let us compare the asymptotic behaviour for solutions of ODEs, as in (2.5), and for solutions of stochastic differential equations, that is, for processes solving

$$
\begin{aligned}
\mathrm{d} \tilde{X}_{t} & =\tilde{b}\left(\tilde{X}_{t}\right) \mathrm{d} t+\tilde{\sigma}\left(\tilde{X}_{t}\right) \mathrm{d} B_{t}, \\
\tilde{X}_{0} & =x,
\end{aligned}
$$

where $B$ is an $m$-dimensional Brownian motion.

Let $\tilde{Y}^{n}$ be defined as

$$
\tilde{Y}_{t}^{n}=\Gamma_{\alpha_{n}}\left(\tilde{X}_{n t}\right), \quad t \in[0,1] .
$$

It easily follows that $\tilde{Y}^{n}$ is again a diffusion process, whose drift $\bar{b}_{n}$ and diffusion coefficient $\bar{\sigma}_{n}$ can be computed by means of Itô's formula and by applying a suitable time-change. As proved in Baldi (1986), under Assumptions 2.1 and 2.2, applied to $\bar{b}_{n}$ and $\bar{\sigma}_{n}$, the sequence $\left\{\tilde{Y}^{n}\right\}_{n}$ is a.s. relatively compact in $\mathscr{C}\left([0,1], \mathbb{R}^{d}\right)$ and the limit points are given by the set $K$ in Theorem 2.5, in which the coefficients $b$ and $\sigma$ have obviously to be replaced by $\bar{b}=\lim _{n \rightarrow \infty} \bar{b}_{n}$ and $\bar{\sigma}=\lim _{n \rightarrow \infty} \bar{\sigma}_{n}$, respectively, for the computation of the rate function $J$.

Therefore, for solutions of both ordinary and stochastic differential equations, a law of the iterated logarithm holds, and whenever the drift and 'diffusion' coefficients of $Y^{n}$ and $\tilde{Y}^{n}$ have the same asymptotic behaviour - that is, $b=\bar{b}$ and $\sigma=\bar{\sigma}$ - the set of the limit points does not change.

The proof of Theorem 2.5 will follow from some preliminary results. In what follows, for $\varphi \in \mathscr{C}\left([0,1], \mathbb{R}^{d}\right)$ and $A \subset \mathscr{C}\left([0,1], \mathbb{R}^{d}\right),\|\varphi\|$ and $d(\varphi, A)$ stand for the supremum norm and the usual distance of $\varphi$ from $A$, respectively:

$$
\|\varphi\|=\sup _{t \in[0,1]}\left|\varphi_{t}\right|, \quad d(\varphi, A)=\inf _{\psi \in A}\|\varphi-\psi\| .
$$

Lemma 2.7. Under Assumptions 2.1 and 2.2, 


$$
\lim _{n \rightarrow \infty} d\left(Y^{n}, K\right)=0 \text { a.s. }
$$

Proof. First of all, we prove that

$$
\lim _{j \rightarrow \infty} d\left(Y^{\left[c^{j}\right]}, K\right)=0 \text { a.s., }
$$

where $c>1$ and $[n]$ stands for the integer part of $n-$ that is, the statement holds for suitable subsequences. By using the first Borel-Cantelli lemma, it is sufficient to prove that, for any $\varepsilon>0$, the series $\sum_{j} P\left(d\left(Y^{\left[c^{j}\right]}, K\right)>\varepsilon\right)$ converges.

Let $K_{\varepsilon}^{\prime}$ be an $\varepsilon$-neighbourhood of $K: K_{\varepsilon}^{\prime}=\{g: d(g, K) \geqslant \varepsilon\}$. Since the functional $J$ is lower semi-continuous and $K$ is compact, it follows that there exists $\delta>0$ such that, for any $\varphi \in K_{\varepsilon}^{\prime}$,

$$
\inf _{\varphi \in K_{\varepsilon}^{\prime}} J(\varphi)>1+2 \delta .
$$

Now, by using the moderate-deviation upper bound given by Theorem 2.3, we have, for large values of $j$,

$$
P\left(Y^{\left[c^{j}\right]} \in K_{\varepsilon}^{\prime}\right) \leqslant \exp \left(-(1-\delta) \ln \ln \left[c^{j}\right]\right) \leqslant \frac{\text { const. }}{(j-1)^{1+\delta}}
$$

because, for any large $j,\left[c^{j}\right] \geqslant c^{j-1}$. Therefore, the series $\sum P\left(Y^{\left[c^{j}\right]} \in K_{\varepsilon}^{\prime}\right)$ converges, so that, for any $c>1, \lim _{j \rightarrow \infty} d\left(Y^{\left[c^{j}\right]}, K\right)=0$ a.s.

Now fix $n$ : for any $c>1$ and $j$ such that $\left[c^{j}\right] \leqslant n \leqslant\left[c^{j+1}\right]$, we can write

$$
\begin{aligned}
d\left(Y^{n}, K\right) & \leqslant d\left(Y^{\left[c^{j}\right]}, K\right)+\left\|Y^{n}-Y^{\left[c^{j}\right]}\right\| \\
& \leqslant d\left(Y^{\left[c^{j}\right]}, K\right)+\left\|\Gamma_{\alpha_{n}} \circ \Gamma_{\left[c^{j}\right]}^{-1}\left(Y^{\left[c^{j}\right]}\right)-Y^{\left[c^{j}\right]}\right\|+\sup _{t \in[0,1]}\left|\Gamma_{\alpha_{n}}\left(X_{\left[c^{j}\right] t}\right)-\Gamma_{\alpha_{n}}\left(X_{n t}\right)\right| .
\end{aligned}
$$

We shall now estimate $d\left(Y^{n}, K\right)$ by proving that it is small enough for large values of $n$. All the upper bounds we shall state have to be considered a.s.

By the first part of this proof, for any $\varepsilon>0$ and $c>1$ there exists $j_{c}$ such that, for $j>j_{c}$,

$$
d\left(Y^{\left[c^{j}\right]}, K\right)<\varepsilon,
$$

so that

$$
d\left(Y^{n}, K\right) \leqslant \varepsilon+\left\|\Gamma_{\alpha_{n}} \circ \Gamma_{\left[c^{j}\right]}^{-1}\left(Y^{\left[c^{j}\right]}\right)-Y^{\left[c^{j}\right]}\right\|+\sup _{t \in[0,1]}\left|\Gamma_{\alpha_{n}}\left(X_{\left[c^{j} t\right.}\right)-\Gamma_{\alpha_{n}}\left(X_{n t}\right)\right| .
$$

Moreover, for any large $j$,

$$
1 \leqslant \frac{\alpha_{n}}{\alpha_{\left[c^{j}\right]}}=\sqrt{\frac{n \ln \ln n}{\left[c^{j}\right] \ln \ln \left[c^{j}\right]}} \leqslant \sqrt{\frac{\left[c^{j+1}\right] \ln \ln \left[c^{j+1}\right]}{\left[c^{j}\right] \ln \ln \left[c^{j}\right]}} \leqslant \sqrt{\frac{\left(c^{j+1}\right) \ln \ln \left(c^{j+1}\right)}{\left(c^{j}-1\right) \ln \ln \left(c^{j}-1\right)}}=: \gamma_{j}
$$

and $\gamma_{j} \rightarrow \sqrt{c}$ as $j \rightarrow \infty$. It then follows that for any $\delta>0$ there exist $c_{1}>1$ and $j_{1}$ such that for any $c \in\left(1, c_{1}\right), j>j_{1}$ and $n \in\left[\left[c^{j}\right],\left[c^{j+1}\right]\right]$, 


$$
\left|\frac{\alpha_{n}}{\alpha_{\left[c^{j}\right]}}-1\right| \leqslant \delta .
$$

By using property (iii) of Assumption 2.1, we can state that for any $\varepsilon>0$ and for any compact $C$ of $\mathbb{R}^{d}$ there exist $c_{1}>1$ and $j_{1}$ such that for any $c \in\left(1, c_{1}\right), j>j_{1}$ and $n \in\left[\left[c^{j}\right],\left[c^{j+1}\right]\right]$,

$$
\sup _{y \in C}\left|\Gamma_{\alpha_{n}} \circ \Gamma_{\left[c^{j}\right]}^{-1}(y)-y\right|<\varepsilon .
$$

Now fix $c \in\left(1, c_{1}\right)$ : since $d\left(Y^{\left[c^{j}\right]}, K\right) \rightarrow 0$ as $j \rightarrow \infty$, there exists $\hat{j}_{1}$ such that, for any $j>\hat{j}_{1}$,

$$
\left\|Y^{\left[c^{j}\right]}\right\| \leqslant 2 \sup _{\varphi \in K}\|\varphi\|=: M
$$

and $M$ is a finite number because $K$ is compact. Therefore, for any $\varepsilon>0$ one can find $c_{1}>1$ such that for any $c \in\left(1, c_{1}\right)$ there exists $i_{1}=\max \left(j_{c}, j_{1}, \hat{j}_{1}\right)$ such that for any $j>i_{1}$ and $n \in\left[\left[c^{j}\right],\left[c^{j+1}\right]\right]$,

$$
\left\|\Gamma_{\alpha_{n}} \circ \Gamma_{\left[c^{j}\right]}^{-1}\left(Y^{\left[c^{j}\right]}\right)-Y^{\left[c^{j}\right]}\right\| \leqslant \sup _{\{y:|y| \leqslant M\}}\left|\Gamma_{\alpha_{n}} \circ \Gamma_{\left[c^{j}\right]}^{-1}(y)-y\right|<\varepsilon
$$

and (16) holds, so that

$$
d\left(Y^{n}, K\right) \leqslant 2 \varepsilon+\sup _{t \in[0,1]}\left(X_{\left[c^{j}\right] t}\right)-\Gamma_{\alpha_{n}}\left(X_{n t}\right) \mid .
$$

We now have to estimate the quantity appearing on the right-hand side of the above inequality.

First of all, since $\alpha_{n} \geqslant \xi_{\left[c^{j}\right]}$, by using condition (ii) of Assumption 2.1 we can write

$$
\begin{aligned}
\sup _{t \in[0,1]}\left|\Gamma_{\alpha_{n}}\left(X_{\left[c^{j}\right] t}\right)-\Gamma_{\alpha_{n}}\left(X_{n t}\right)\right| & \leqslant \sup _{t \in[0,1]}\left|\Gamma_{\left[c^{j}\right]}\left(X_{\left[c^{j}\right] t}\right)-\Gamma_{\left[c^{j}\right]}\left(X_{n t}\right)\right| \\
& \leqslant \sup _{0 \leqslant u \leqslant 1,0 \leqslant s \leqslant \frac{\left[c^{j}\right]}{\left[c^{j+1}\right]} u}\left|\Gamma_{\left[c^{j}\right]}\left(X_{\left[c^{j+1}\right] u}\right)-\Gamma_{\left[c^{j+1}\right]}\left(X_{\left[c^{j+1}\right] s}\right)\right|,
\end{aligned}
$$

where the latter inequality results from setting $n t=\left[c^{j+1}\right] u$ and $\left[c^{j}\right] t=\left[c^{j+1}\right] s$. By adding and subtracting $Y_{u}^{\left[c^{j+1}\right]}$ and $Y_{s}^{\left[c^{j+1}\right]}$, we obtain, for any $n \in\left[\left[c^{j}\right],\left[c^{j+1}\right]\right]$,

$$
\begin{aligned}
& \sup _{t \in[0,1]}\left|\Gamma_{\alpha_{n}}\left(X_{\left[c^{j}\right] t}\right)-\Gamma_{\alpha_{n}}\left(X_{n t}\right)\right| \\
& \qquad 2\left\|\Gamma_{\left[c^{j}\right]} \circ \Gamma_{\left[c^{j+1}\right]}^{-1}\left(Y^{\left[c^{j+1}\right]}\right)-Y^{\left[c^{j+1}\right]}\right\|+\sup _{0 \leqslant u \leqslant 1,0 \leqslant s \leqslant\left[c_{\left[c^{j+1}\right]} u\right.}\left|Y_{u}^{\left[c^{j+1}\right]}-Y_{s}^{\left[c^{j+1}\right]}\right| .
\end{aligned}
$$

By using arguments similar to those previously developed, involving property (iii) of Assumption 2.1, we can find $c_{2}>1$ and $i_{2}$ such that for any $c \in\left(1, c_{2}\right)$ and $j>i_{2}$,

$$
2\left\|\Gamma_{\left[c^{j}\right]} \circ \Gamma_{\left[c^{j+1}\right]}^{-1}\left(Y^{\left[c^{j+1}\right]}\right)-Y^{\left[c^{j+1}\right]}\right\|<2 \varepsilon,
$$

so that for $c \in\left(1, \min \left(c_{1}, c_{2}\right)\right)$ and $j>\max \left(i_{1}, i_{2}\right)$, 


$$
d\left(Y^{n}, K\right) \leqslant 4 \varepsilon+\sup _{0 \leqslant u \leqslant 1,0 \leqslant s \leqslant \frac{\left[c^{j}\right]}{\left[c^{j+1}\right]} u}\left|Y_{u}^{\left[c^{j+1}\right]}-Y_{s}^{\left[c^{j+1}\right]}\right| .
$$

Now, since $d\left(Y^{\left[c^{j+1}\right]}, K\right) \rightarrow 0$ as $j \rightarrow \infty$, there exists $i_{3}$ such that, for any $j>i_{3}$,

$$
d\left(Y^{\left[c^{j+1}\right]}, K\right)<\varepsilon .
$$

Therefore, for any such value of $j$ there exists a path $g \in K$ such that

$$
\left\|Y^{\left[c^{j+1}\right]}-g\right\|<2 \varepsilon .
$$

Thus, for any $j>\max \left(i_{1}, i_{2}, i_{3}\right)$, we can write

$$
d\left(Y^{n}, K\right)<6 \varepsilon+\sup _{0 \leqslant u \leqslant 1,0 \leqslant s \leqslant \frac{\left[c^{j}\right]}{\left[c^{j+1}\right]} u}|g(u)-g(s)| .
$$

Finally, since $0 \leqslant u-s \leqslant 1-\left[c^{j}\right] /\left[c^{j+1}\right] \leqslant 1-1 / c$, and $K$ is a compact subset of $\mathscr{C}\left([0,1], \mathbb{R}^{d}\right)$, by the Ascoli-Arzelà theorem there exists $c_{3}>1$ such that, for any $c \in\left(1, c_{3}\right)$,

$$
\sup _{g \in K} \sup _{0 \leqslant u \leqslant 1,0 \leqslant s \leqslant \frac{\left[c^{j}\right]}{\left[c^{j+1}\right]} u}|g(u)-g(s)|<\varepsilon .
$$

Thus, for any $\varepsilon>0$ we have found a constant $c_{0}=\min \left(c_{1}, c_{2}, c_{3}\right)>1$ and an integer $j_{0}=\max \left(i_{1}, i_{2}, i_{3}\right)$ such that if $c \in\left(1, c_{0}\right), j>j_{0}$ and $n \in\left[\left[c^{j}\right],\left[c^{j+1}\right]\right]$, then

$$
d\left(Y^{n}, K\right)<7 \varepsilon,
$$

so that the statement holds.

In order to prove Theorem 2.5, we also need the following result concerning a sort of functional law of the iterated logarithm for standard sums of random variables, which will be used in order to show that $K$ is the set of the limit points for $\left\{Y^{n}\right\}_{n}$. It is well known in the literature that, for the polygon $S^{n}$ defined in (6), $\left\{(1 / \sqrt{n \ln \ln n}) S^{n}\right\}_{n}$ is a.s. relatively compact in $\mathscr{C}\left([0,1], \mathbb{R}^{m}\right)$ and the set of its limit points is given by

$$
C=\{h: v(h) \leqslant 1\} \equiv\left\{h \in \mathscr{C O}_{0}^{m}: \frac{1}{2} \int_{0}^{1}\left|h^{\prime}(s)\right|^{2} \mathrm{~d} s \leqslant 1\right\}
$$

(see, for example, Strassen 1964). In particular, for any $h \in C$ and $\delta>0$,

$$
P\left(\left\|\frac{1}{\sqrt{n \ln \ln n}} S^{n}-h\right\| \leqslant \delta \text { infinitely often }\right)=1 .
$$

The next lemma shows that any $h \in C$ is the limit point of a suitable subsequence of $\left\{(1 / \sqrt{n \ln \ln n}) S^{n}\right\}_{n}$ :

Lemma 2.8. For any $h \in C$ such that $\frac{1}{2} \int_{0}^{1}\left|h^{\prime}(s)\right|^{2} \mathrm{~d} s<1$ and $\delta>0$, an integer $c>1$ exists such that 


$$
P\left(\left\|\frac{1}{\sqrt{c^{2 j} \ln \ln c^{2 j}}} S^{c^{2 j}}-h\right\| \leqslant \delta \text { i.o. }\right)=1 .
$$

Proof. Fix an integer $c>0$, and let $T_{i}: \mathscr{C}\left([0,1], \mathbb{R}^{m}\right) \rightarrow \mathscr{C}\left([0,1], \mathbb{R}^{m}\right), i=1,2,3$, be defined as

$$
\begin{aligned}
& T_{1} g(t)=\left\{\begin{array}{ll}
g(t) & \text { if } t \in\left[0, \frac{1}{c}\right], \\
g\left(\frac{1}{c}\right) & \text { if } t \in\left(\frac{1}{c}, 1\right],
\end{array} \quad T_{2} g(t)= \begin{cases}g\left(\frac{1}{c}\right) & \text { if } t \in\left[0, \frac{1}{c}\right], \\
g(t) & \text { if } t \in\left(\frac{1}{c}, 1\right],\end{cases} \right. \\
& T_{3} g(t)= \begin{cases}g\left(\frac{1}{c^{2}}\right) & \text { if } t \in\left[0, \frac{1}{c}\right], \\
g\left(\frac{t}{c}\right) & \text { if } t \in\left(\frac{1}{c}, 1\right] .\end{cases}
\end{aligned}
$$

It follows that:

(a) $\left(T_{1}+T_{2}\right) g(t)=g(t)+g(1 / c)$, thus $g(t)=\left(T_{1}+T_{2}\right) g(t)-g(1 / c)$;

(b) $|g(1 / c)| \leqslant\left\|T_{1} g\right\|:\left\|T_{1} g\right\|=\sup _{t \in[0,1 / c]}|g(t)| \geqslant|g(1 / c)|$;

(c) $\left\|T_{3} g\right\| \leqslant\left\|T_{1} g\right\|:\left\|T_{3} g\right\|=\sup _{t \in[1 / c, 1]}|g(t / c)|=\sup _{t \in\left[1 / c^{2}, 1 / c\right]}|g(t)| \leqslant \sup _{t \in[0,1 / c]}$ $|g(t)|=\left\|T_{1} g\right\|$.

Let us write

$$
\xi^{j}(t)=\frac{1}{\sqrt{c^{2 j} \ln \ln c^{2 j}}} S_{t}^{c^{2 j}}
$$

Using $(a),(b),(c)$, we have

$$
\begin{aligned}
\left\|\xi^{j}-h\right\| & \leqslant\left\|\left(T_{1}+T_{2}\right) \xi^{j}-\left(T_{1}+T_{2}\right) h\right\|+\left|\xi^{j}\left(\frac{1}{c}\right)-h\left(\frac{1}{c}\right)\right| \\
& \leqslant\left\|T_{1} \xi^{j}-T_{1} h\right\|+\left|\xi^{j}\left(\frac{1}{c}\right)-h\left(\frac{1}{c}\right)\right|+\left\|T_{2} \xi^{j}-T_{2} h\right\| \\
& \leqslant\left\|T_{1} \xi^{j}\right\|+\left\|T_{1} h\right\|+\left|\xi^{j}\left(\frac{1}{c}\right)\right|+\left|h\left(\frac{1}{c}\right)\right|+\left\|\left(T_{2}-T_{3}\right) \xi^{j}-T_{2} h\right\|+\left\|T_{3} \xi^{j}\right\| \\
& \leqslant 3\left\|T_{1} \xi^{j}\right\|+2\left\|T_{1} h\right\|+\left\|\left(T_{2}-T_{3}\right) \xi^{j}-T_{2} h\right\| .
\end{aligned}
$$


Now

$$
\left\|T_{1} h\right\|=\sup _{t \in\left[0, \frac{1}{c}\right]}|h(t)|,
$$

where $h(0)=0$ and $h$ is continuous: there exists $c_{1, \delta}>1$ such that, for any $c>c_{1, \delta}$,

$$
2\left\|T_{1} h\right\|<\frac{\delta}{3} \text {. }
$$

Thus, for any $c>c_{1, \delta}$,

$$
\left\|\xi^{j}-h\right\| \leqslant \frac{\delta}{3}+3\left\|T_{1} \xi^{j}\right\|+\left\|\left(T_{2}-T_{3}\right) \xi^{j}-T_{2} h\right\| .
$$

Let us first consider the term $3\left\|T_{1} \xi^{j}\right\|=3 \sup _{t \in[0,1 / c]}\left|\xi^{j}(t)\right|$. Set

$$
F_{\delta}=\left\{g \in \mathscr{C}: 3\left\|T_{1} g\right\| \geqslant \frac{\delta}{3}\right\}
$$

so that $3\left\|T_{1} \xi^{j}\right\| \geqslant \delta / 3$ if and only if $\xi^{j} \in F_{\delta} . F_{\delta}$ is a closed subset and, by Remark 2.4(i), $\xi^{j}$ verifies a moderate-deviation principle at speed $\left(c^{2 j} \ln \ln c^{2 j}\right) / c^{2 j}=\ln \ln c^{2 j}$, with rate function $v$ defined in (12), so that we can use the upper bound applied to $F_{\delta}$. Notice that if $g \in F_{\delta}$ then for $t \leqslant 1 / c$, we have

$$
|g(t)|=\left|\int_{0}^{t} g^{\prime}(s) \mathrm{d} s\right| \leqslant\left(\int_{0}^{t}\left|g^{\prime}(s)\right|^{2} d s\right)^{1 / 2}\left(\int_{0}^{1 / c} d s\right)^{1 / 2} \leqslant \frac{1}{\sqrt{c}} \sqrt{2 v(g)}
$$

so that

$$
v(g) \geqslant \frac{c}{2}|g(t)|^{2} \geqslant \frac{c \cdot \delta^{2}}{2 \cdot 81}
$$

and

$$
\inf _{g \in F_{\delta}} v(g) \geqslant \frac{c \cdot \delta^{2}}{2 \cdot 81} \text {. }
$$

It follows that there exists $c_{2, \delta}>1$ such that for any integer $c>c_{2, \delta}$ we have $c \cdot \delta^{2} /(2 \cdot 81)>2$, and thus

$$
\inf _{g \in F_{\delta}} v(g) \geqslant 2 .
$$

For such values of $c$, by using the moderate-deviation upper estimate, we obtain, for large values of $j$,

$$
\begin{aligned}
P\left(3\left\|T_{1} \xi^{j}\right\| \geqslant \frac{\delta}{3}\right) & =P\left(\xi^{j} \in F_{\delta}\right) \leqslant \exp \left(-\left(\ln \ln c^{2 j} \cdot \inf _{g \in F_{\delta}} v(g)\right)\right. \\
& \leqslant \exp \left(-\left(\ln \ln c^{2 j}\right) \frac{3}{2}\right)=\frac{\text { const. }}{j^{3 / 2}} .
\end{aligned}
$$


It then follows that the associated series converges and

$$
P\left(3\left\|T_{1} \xi^{j}\right\| \geqslant \frac{\delta}{3} \text { i.o. }\right)=0 .
$$

Let us return to (17): if $c_{\delta}=\max \left(c_{1, \delta}, c_{2, \delta}\right)$ then for $c>c_{\delta}>1, c$ integer, we can state that

$$
\left\|\xi^{j}-h\right\| \leqslant \frac{2}{3} \delta+\left\|\left(T_{1}-T_{2}\right) \xi^{j}-T_{2} h\right\| \text { a.s. },
$$

for any $j$ large. It remains to prove that, for $c>c_{\delta}$,

$$
P\left(\left\|\left(T_{1}-T_{2}\right) \xi^{j}-T_{2} h\right\|<\frac{\varepsilon}{3} \text { i.o. }\right)=1 .
$$

We will make use of the second Borel-Cantelli lemma, which can be applied because the random variables $\left\{\left(T_{2}-T_{3}\right) \xi^{j}\right\}_{j}$ are independent. In fact,

$$
\left(T_{2}-T_{3}\right) \xi_{t}^{j}= \begin{cases}\frac{1}{\sqrt{c^{2 j} \ln \ln c^{2 j}}} \cdot\left(S_{1 / c}^{c^{2 j}}-S_{1 / c^{2}}^{c^{2 j}}\right) & \text { if } t \leqslant \frac{1}{c}, \\ \frac{1}{\sqrt{c^{2 j} \ln \ln c^{2 j}}} \cdot\left(S_{t}^{c^{2 j}}-S_{t / c}^{c^{2 j}}\right) & \text { if } t>\frac{1}{c} .\end{cases}
$$

Now, if $t \geqslant 1 / c$,

$$
\begin{aligned}
S_{t}^{c^{2 j}}-S_{t / c}^{c^{2 j}} & =\sum_{k=1}^{\left[c^{2 j} t\right]} Z^{k}+\left(c^{2 j} t-\left[c^{2 j} t\right]\right) Z^{\left[c^{2 j} t\right]+1}-\sum_{k=1}^{\left[c^{2 j-1} t\right]} Z^{k}-\left(c^{2 j-1} t-\left[c^{2 j-1} t\right]\right) Z^{\left[c^{2 j-1} t\right]+1} \\
& =-\left(c^{2 j-1} t-\left[c^{2 j-1} t\right]\right) Z^{\left[c^{2 j-1} t\right]+1}+\sum_{k=\left[c^{2 j-1} t\right]+1}^{\left[c^{2 j} t\right]} Z^{k}+\left(c^{2 j} t-\left[c^{2 j} t\right]\right) Z^{\left[c^{2 j} t\right]+1} .
\end{aligned}
$$

Thus, the random variable $\left(T_{2}-T_{3}\right) \xi^{j}$ is $\sigma\left(Z^{c^{2 j-2}+1}, \ldots, Z^{c^{2 j}}\right)$-measurable: since $\left(Z^{n}\right)_{n}$ is a sequence of independent random variables, it then follows that $\left(T_{2}-T_{3}\right) \xi^{j}$ are independent, as $j$ varies. Thus, if

$$
\left.\sum_{k} P\left(\| T_{2}-T_{3}\right) \xi^{j}-T_{2} h \|<\frac{\delta}{3}\right)=+\infty
$$

it would follow that

$$
P\left(\left\|\left(T_{2}-T_{3}\right) \xi^{j}-T_{2} h\right\|<\frac{\delta}{3} \text { i.o. }\right)=1 .
$$

Therefore, we have only to show that (18) holds.

Set

$$
G_{\delta}=\left\{g: g(0)=0 \text { and }\left\|\left(T_{2}-T_{3}\right) g-T_{2} h\right\|<\frac{\delta}{3}\right\}
$$


so that $P\left(\left\|\left(T_{2}-T_{3}\right) \xi^{j}-T_{2} h\right\|<\delta / 3\right)=P\left(\xi^{j} \in G_{\delta}\right)$. Since $G_{\delta}$ is an open set, the moderatedeviation lower bound in Remark 2.4 gives, for any large $j$.

$$
P\left(\xi^{j} \in G_{\delta}\right) \geqslant \exp \left(-\ln \ln c^{2 j} \cdot \inf _{g \in G_{\delta}} v(g)\right),
$$

Now, having chosen $c>c_{\delta} \geqslant c_{1, \delta}$, it follows that $h \in G_{\delta}$ :

$$
\left\|\left(T_{2}-T_{3}\right) h-T_{2} h\right\|=\left\|T_{3} h\right\| \leqslant\left\|T_{1} h\right\| \leqslant \frac{\delta}{6}<\frac{\delta}{3} .
$$

Thus, $\inf _{g \in G} v(g) \leqslant v(h)<1$ and

$$
P\left(\xi^{j} \in G\right) \geqslant \exp \left(-\ln \ln c^{2 j}\right)=\frac{\text { const. }}{2 j} .
$$

Since the associated series diverges, statement (18) holds.

With these preliminaries completed, we can now prove our earlier result.

Proof of Theorem 2.5. Lemma 2.7 enables us easily to prove that the sequence $\left\{Y^{n}\right\}_{n}$ is a.s. totally bounded in the space of continuous paths, so that it is relatively compact. In fact, since $K$ is compact, for any $\varepsilon>0$ there exist a finite number of paths $\varphi_{1}, \ldots, \varphi_{N}$ such that

$$
K \subset \bigcup_{i=1}^{N} B\left(\varphi_{i}, \frac{\varepsilon}{2}\right)
$$

where $B\left(\varphi_{i}, \varepsilon / 2\right)$ is the ball centred in $\phi_{i}$ of radius $\varepsilon / 2$ in the space $\mathscr{C}\left([0,1], \mathbb{R}^{d}\right)$. By Lemma 2.7, there exists a.s. a value $n_{0}$ such that, for any $n>n_{0}$,

$$
d\left(Y^{n}, K\right)<\frac{\varepsilon}{2}
$$

so that

$$
\left\{Y^{n}\right\}_{n>n_{0}} \subset \bigcup_{i=1}^{N} B\left(\varphi_{i}, \varepsilon\right) .
$$

Since obviously $\left\{Y^{n}\right\}_{n \leqslant n_{0}} \subset \bigcup_{i=1}^{n_{0}} B\left(Y^{n}, \varepsilon\right)$, it follows that

$$
\left\{Y^{n}\right\}_{n} \subset\left(\bigcup_{i=1}^{n_{0}} B\left(Y^{n}, \varepsilon\right)\right) \cup\left(\bigcup_{i=1}^{N} B\left(\varphi_{i}, \varepsilon\right)\right),
$$

that is, $\left\{Y^{n}\right\}_{n}$ is a.s. totally bounded in $\mathscr{C}\left([0,1], \mathbb{R}^{d}\right)$ and therefore is relatively compact in $\mathscr{C}\left([0,1], \mathbb{R}^{d}\right)$.

In order to prove that $K$ is the set of all the limit points, it suffices to prove that for any $\varphi \in K$ such that $J(\varphi)<1$,

$$
P\left(\left\|Y^{n}-\varphi\right\|<\varepsilon \text { i.o. }\right)=1,
$$

where $\varepsilon>0$. 
Since, by Remark 2.4(ii), there exists $h \in \mathscr{C O}_{0}^{m}$ such that $\frac{1}{2} \int_{0}^{1}\left|h^{\prime}(s)\right|^{2} \mathrm{~d} s<1$ and $\varphi=$ $F_{x}(h)$, by using Theorem 2.3 we can state that there exists $\delta>0$ such that

$$
\limsup _{n \rightarrow \infty} \frac{1}{\ln \ln n} \ln P\left(\left\|Y^{n}-\varphi\right\|<\varepsilon,\left\|\frac{1}{\sqrt{n \ln \ln n}} S^{n}-h\right\|>\delta\right) \leqslant-3 .
$$

By Lemma 2.8, there exists an integer $c>1$, such that

$$
P\left(\left\|\frac{1}{\sqrt{c^{2 j} \ln \ln c^{2 j}}} S^{c^{2 j}}-h\right\| \leqslant \delta \text { i.o. }\right)=1 .
$$

Setting

$$
G_{j}=\left\{\left\|Y^{c^{2 j}}-\varphi\right\|<\varepsilon\right\}, \quad H_{j}=\left\{\left\|\frac{1}{\sqrt{c^{2 j} \ln \ln c^{2 j}}} S_{t}^{c^{2 j}}-h\right\|<\delta\right\},
$$

then $P\left(H_{j}\right.$ i.o. $)=1$, and by $(20)$ we obtain, for any large $j$,

$$
P\left(H_{j} \cap G_{j}^{C}\right) \leqslant \exp \left(-2 \ln \ln c^{2 j}\right)=\frac{\text { const. }}{(2 j)^{2}},
$$

so that

$$
P\left(H_{j} \cap G_{j}^{C} \text { i.o. }\right)=0 .
$$

It then follows that $P\left(G_{j}\right.$ i.o. $)=1$ :

$$
P\left(G_{j} \text { i.o. }\right) \geqslant P\left(H_{j} \cap G_{j} \text { i.o. }\right)+P\left(H_{j} \cap G_{j}^{C} \text { i.o. }\right) \geqslant P\left(H_{j} \text { i.o. }\right)=1
$$

and (19) holds.

Remark 2.9. Theorem 2.5 still holds if the covariance matrix $\Lambda$ of the random variable $Z^{1}$ is not the identity, subject to a modification of the representation $K$ of the limit points. Indeed, if $r(\leqslant m)$ is the rank of $\Lambda$, then one can build a $m \times r$ matrix $M$ such that $\Lambda=M M^{*}$, and a sequence $\left\{\tilde{Z}^{k}\right\}_{k}$ of i.i.d. $\mathbb{R}^{r}$-valued random variables with the $r \times r$ identity as covariance matrix, so that $Z^{k}=M \tilde{Z}^{k}$. Therefore, the processes $X$ and $Y^{n}=\Gamma_{\alpha_{n}}\left(X_{n}\right.$.) solve the differential equations

$$
\dot{X}_{t}=b\left(X_{t}\right)+\hat{\sigma}\left(Y_{t}^{n}\right) M \dot{\tilde{S}}(t), \quad \dot{Y}_{t}^{n}=b_{n}\left(Y_{t}^{n}\right)+\sigma_{n}\left(Y_{t}^{n}\right) M \frac{1}{\alpha_{n}} \dot{\tilde{S}}^{n}(t)
$$

respectively, where $\tilde{S}$ and $\tilde{S}^{n}$ are the process defined in (3) and (6) respectively, by replacing $Z^{k}$ with $\tilde{Z}^{k}$. Thus, if $b_{n}$ and $\sigma_{n}$ satisfy the conditions required in Theorem 2.5 , these are verified by $b_{n}$ and $\sigma_{n} \cdot M$ too, and one can apply Theorem 2.5 in order to find the asymptotic behaviour of $\left\{Y^{n}\right\}_{n}$. Notice that in the computation of the rate function $J$, the matrix field $\sigma=\lim _{n \rightarrow \infty} \sigma_{n}$ has to be replaced by $\lim _{n \rightarrow \infty} \sigma_{n} \cdot M$. 


\section{Law of the iterated logarithm on nilpotent Lie groups}

In this section we apply Theorem 2.5 in order to obtain a law of the iterated logarithm for random walks on a simply connected, graded, nilpotent Lie group $G$.

Let $\mathbb{G}$ denote the Lie algebra associated with $G$, which we suppose to be graded, as in the Introduction: there exist $l$ vector spaces $V_{1}, \ldots V_{l}$ such that

$$
\mathbb{G}=V_{1} \oplus \cdots \oplus V_{l} \quad \text { and }\left[V_{i}, V_{j}\right] \subseteq V_{i+j},
$$

where $[\cdot, \cdot]$ denotes the bracket operation on $\mathbb{G}$ and $V_{i+j}=\{0\}$ if $i+j>l$. Let $\left\{e_{1}, \ldots, e_{d}\right\}$ be a basis of $\mathbb{b}$ which is adapted to the decomposition above: there exist positive integers $0=i_{0}<i_{1}<i_{2}<\ldots<i_{l}$ such that $V_{k}$ is spanned by $\left\{e_{i_{k-1}+1}, \ldots, e_{i_{k}}\right\}$, as $k=1, \ldots, l$. In particular, $i_{1}$ denotes the dimension of $V_{1}$. Let $\chi_{i}$ stand for the left-invariant vector field associated with $e_{i}$ : for any smooth function $f$,

$$
\chi_{i} f(y)=\left.\frac{\mathrm{d}}{\mathrm{d} t} f\left(y \circ t e_{i}\right)\right|_{t=0}=\sum_{j=1}^{d} \chi_{i}^{j}(y) \frac{\partial f}{\partial y_{j}}(y) .
$$

Let $\hat{\sigma}(y)=\left[\chi_{1}(y) \ldots \chi_{d}(y)\right]$ be the matrix with $i$ th column vector $\chi_{i}(y)$. The crucial remark is that for a vector $a \in \mathbb{R}^{d} \simeq \mathfrak{G}$, the solution of

$$
\begin{aligned}
& \dot{x}_{t}=\hat{\sigma}\left(x_{t}\right) a, \\
& x_{0}=g
\end{aligned}
$$

is given by

$$
x_{t}=g \circ(t a) .
$$

Indeed, it is well known that $x_{t}=g \circ \operatorname{Exp}(t a)$ is the solution of (23), Exp $: \mathbb{G} \rightarrow G$ being the exponential mapping, which allows $G$ to be identified with its Lie algebra $\mathfrak{E}$, so that (24) actually holds.

Moreover, the product on $G$ is related to the structure of $\mathbb{G}$ by the Campbell-Hausdorff formula:

$$
x \circ y=x+y+\frac{1}{2}[x, y]+\frac{1}{12}[x,[x, y]]+\frac{1}{12}[y,[y, x]]+\ldots
$$

Therefore, it turns out that by (21) and (25),

$$
\chi_{i}(y) \in V_{i} \oplus \ldots \oplus V_{l} .
$$

Consider now a sequence of i.i.d. random variables $\left\{Z^{j}\right\}_{j}$ taking values on $G$. Suppose that each $Z^{j}$ is centred on $G$ - that is, $\mathrm{E}\left[P_{1} Z^{j}\right]=0$, where $P_{1}$ is the projection map on $V_{1}$ - and that the covariance matrix is the identity. In the proofs of the results of the preceding section, which we shall need to use, the hypothesis that the $Z^{j}$ are (fully) centred is crucial in order for Theorem 2.3 to be applied, that is, for the validity of the moderate-deviation estimates. Thus, for any $j$, let us consider the random variable

$$
\tilde{Z}^{j}=Z^{j}-\mathrm{E}\left[Z^{j}\right]
$$


which can be obviously considered as a centred random variable on $\mathbb{R}^{d} \simeq \mathbb{G}$. We define the 'standard' polygon $S_{t}$ and $\tilde{S}_{t}$, associated with $\left\{Z^{j}\right\}_{j}$ and $\left\{\tilde{Z}^{j}\right\}_{j}$ respectively, as in (3), that is,

$$
S_{t} \sum_{k=1}^{[t]} Z^{k}+(t-[t]) Z^{[t]+1} \quad \text { and } \quad \tilde{S}_{t}=\sum_{k=1}^{[t]} \tilde{Z}^{k}+(t-[t]) \tilde{Z}^{[t]+1}=S_{t}-t \mathrm{E}\left[Z^{1}\right] \text {, }
$$

where $S_{0}=\tilde{S}_{0}=0$. Let $X$ denote the solution of the (ordinary) differential equation

$$
\begin{aligned}
\dot{X}_{t} & =\hat{\sigma}\left(X_{t}\right) \dot{S}_{t}, \quad t>0, \\
X_{0} & =0 .
\end{aligned}
$$

Since $\dot{S}_{t}=Z^{1}$ as $t \in(0,1)$, by (23) and (24) we have

$$
X_{1}=0 \circ Z^{1}=Z^{1} \text {. }
$$

By recurrence one easily obtains $X_{k}=Z^{1} \circ \ldots \circ Z^{k}$ and, as $k=n$,

$$
X_{n}=Z^{1} \circ \ldots \circ Z^{n}=T^{n} .
$$

Notice that, by using the polygon $\tilde{S}$, it turns out that $X$ also solves the ODE

$$
\begin{aligned}
\dot{X}_{t} & =\hat{\sigma}\left(X_{t}\right) \mathrm{E}\left[Z^{1}\right]+\hat{\sigma}\left(X_{t}\right) \dot{\tilde{S}}_{t}, \quad t>0, \\
X_{0} & =0 .
\end{aligned}
$$

For $\alpha>0$, consider the map

$$
\Gamma_{\alpha}=D_{1 / \alpha} \equiv D_{\alpha}^{-1}
$$

where $D_{\alpha}$ is the dilatation defined in (1). It is immediate to check that $\Gamma_{\alpha}$ satisfies (8) and the process $Y^{n}$, defined as in (5), becomes

$$
Y_{t}^{n}=D_{1 / \sqrt{n \ln \ln n}} X_{n t}, \quad t \in[0,1] .
$$

Thus, as $k=1,2, \ldots, n$, we have $Y_{k / n}^{n}=D_{1 / \sqrt{n \ln \ln n}} T^{k}$, so that

$$
Y_{1}^{n}=D_{1 / \sqrt{n \ln \ln n}} T^{n} .
$$

Moreover, using the representation in (28), $Y^{n}$ solves the differential equation

$$
\begin{aligned}
\dot{Y}_{t}^{n} & =b_{n}\left(Y^{n}\right)+\sigma_{n}\left(Y_{t}^{n}\right) \frac{1}{\alpha_{n}} \dot{\tilde{S}}_{t}^{n}, \\
Y_{0}^{n} & =0,
\end{aligned}
$$

where $\alpha_{n}=\sqrt{n \ln \ln n}$ and, by (10) and (11),

$$
\begin{aligned}
& \sigma_{n}(y)=\left.\alpha_{n} \nabla D_{1 / \alpha_{n}}(z) \hat{\sigma}(z)\right|_{z=D_{1 / \alpha_{n}}^{-1}(y)}=\left.a_{n} D_{1 / \alpha_{n}} \cdot \hat{\sigma}(z)\right|_{z=D_{1 / \alpha_{n}}^{-1}(y),} \\
& b_{n}(y)=\frac{n}{\alpha_{n}} \sigma_{n}(y) .
\end{aligned}
$$

In order to study the asymptotic behaviour of $\left\{D_{1 / \sqrt{n \ln \ln n}} T^{n}\right\}_{n}$, we consider first the 
sequence $\left\{Y^{n}\right\}_{n}$, since by applying Theorem 2.5 one almost immediately obtains the following result, which can be interpreted as a functional law of the iterated logarithm on simply connected, graded, nilpotent Lie groups.

Theorem 3.1. The sequence $\left\{Y^{n}\right\}_{n}$ defined in (29) is a.s. relatively compact on $\mathscr{C}([0,1], G)$, and the set of its limit points is

$$
K=\{x \in \mathscr{C}([0,1], G): J(x) \leqslant 1\},
$$

with the rate function $J$ as in (13), where $b=0$ and $\sigma$ is the matrix field having $\chi_{i}$ as its ith column for $i=1, \ldots, i_{1}$, the rest being equal to 0 :

$$
\sigma(y)=\left[\chi_{1}(y) \ldots \chi_{i_{1}}(y) 0 \ldots 0\right] .
$$

Proof. We have only to prove that Assumption 2.2 holds. We first study the convergence of $b_{n}$ and $\sigma_{n}$, as $n \rightarrow \infty$.

Let us remark that if $e_{i} \in V_{k}$, then for every $\gamma \in \mathbb{R}^{+}$the vector field $y \mapsto \chi_{i}(y)=$ $\left(\chi_{i}^{j}(y)\right)_{j}$ satisfies the relation

$$
D_{\gamma} \chi_{i}(y)=\gamma^{k} \chi_{i}\left(D_{\gamma}(y)\right)
$$

(see Baldi 1986, Lemma 4.2, for a complete proof). Denoting by $k(i)$ the index $k$ such that $e_{i} \in V_{k}$ and recalling that $\hat{\sigma}(y)=\left[\chi_{1}(y) \ldots \chi_{d}(y)\right]$, by using (31) we can state that

$$
D_{\gamma} \cdot \hat{\sigma}(y)=\left[\gamma^{k(1)} \cdot \chi_{1}(y) \gamma^{k(2)} \cdot \chi_{2}(y) \ldots \gamma^{k(d)} \cdot \chi_{d}(y)\right] .
$$

Therefore, the matrix $\sigma_{n}$ is obtained by multiplying the $i$ th column of $\hat{\sigma}$ by $\alpha_{n}^{-k(i)+1}$ :

$$
\sigma_{n}(y)=\left[\chi_{1}(y) \ldots \chi_{i_{1}}(y) \frac{1}{\alpha_{n}} \chi_{i_{1}+1}(y) \ldots \frac{1}{\alpha_{n}^{l-1}} \chi_{d}(y)\right]
$$

so that $\sigma_{n}(y)$ converges, uniformly on the compact sets, to the matrix field $\sigma$ which has its first $i_{1}$ columns equal to the corresponding columns of $\hat{\sigma}$, and the rest set equal to 0 :

$$
\lim _{n \rightarrow \infty} \sigma_{n}(y)=\sigma(y)=\left[\chi_{1}(y) \ldots \chi_{i_{1}}(y) 0 \ldots 0\right] .
$$

Concerning $b_{n}$, we can write

$$
b_{n}(y)=\frac{n}{\alpha_{n}}\left[\chi_{1}(y) \ldots \chi_{i_{1}}(y) \frac{1}{\alpha_{n}} \chi_{i_{1}+1}(y) \ldots \frac{1}{\alpha_{n}^{l-1}} \chi_{d}(y)\right] \cdot \mathrm{E}\left[Z^{1}\right] .
$$

Now, since $\mathrm{E}\left[Z_{i}^{1}\right]=0$ for $i \leqslant i_{1}$, we have

$$
\left(b_{n}(y)\right)_{j}=\sum_{i=i_{1}+1}^{d} \frac{n}{\alpha_{n}^{k(i)}} \chi_{i}^{j}(y) \mathrm{E}\left[Z_{i}^{1}\right], \quad 1 \leqslant j \leqslant d,
$$

and, by (26), as $i>i_{1}$ we have that $\chi_{i}^{j}(y)=0$ for any $j \leqslant i_{1}$, so that 


$$
\left(b_{n}(y)\right)_{j}= \begin{cases}0 & \text { if } j \leqslant i_{1}, \\ \sum_{i=i_{1}+1}^{d} \frac{n}{\alpha_{n}^{k(i)}} \chi_{i}^{j}(y) \mathrm{E}\left[Z_{i}^{1}\right] & \text { if } j>i_{1} .\end{cases}
$$

Bearing in mind that, for any $i>i_{1}, k(i) \geqslant 2$, it follows that

$$
\lim _{n \rightarrow \infty} b_{n}(y)=0,
$$

and the convergence is uniform on the compact sets.

Moreover, since the vector fields $\chi_{i}$ are $\mathscr{C}^{\infty}$, all the requirements in Assumption 2.2 concerning Lipschitz conditions turn out to be fulfilled. Finally, as has been proved in Lemma 4.3 of Baldi (1986), the Cauchy problem in Assumption 2.2(iv) has a unique solution, so that Assumption 2.2 holds. Thus, Theorem 2.5 can be applied and the statement holds.

Now let

$$
\Psi: \mathscr{C}([0,1], G) \rightarrow G, \quad \Psi(x)=x_{1},
$$

so that, by (30),

$$
\Psi\left(Y^{n}\right)=D_{1 / \sqrt{n \ln \ln n}} T^{n} .
$$

Such a representation allows a law of the iterated logarithm to be stated for random walks on simply connected, graded, nilpotent Lie groups as follows:

Theorem 3.2. The set of the a.s. limit points of the sequence $\left\{D_{1 / \sqrt{n \ln \ln n}} T^{n}\right\}_{n}$ is given by

$$
K_{1}=\Psi(K)=\{g: I(g) \leqslant 1\},
$$

where

$$
I(g)=\inf _{x: g=x_{1}} J(x)
$$

Proof. Since $\Psi$ is a continuous map, the relative compactness of the sequence defined by $D_{1 \sqrt{n \ln \ln n}} T^{n}=Y_{1}^{n}=\Psi\left(Y^{n}\right)$ immediately follows and the set of its limit points is given by $K_{1}=\Psi(K)$. Moreover, setting

$$
I(g)=\inf _{x: g=x_{1}} J(x),
$$

$I$ turns out to be the rate function associated with the moderate-deviation principle satisfied by $\left\{D_{1 \sqrt{n \ln \ln n}} T^{n}\right\}_{n}$ (see Baldi and Caramellino 1999, Theorem 6), so that $I$ is lower semicontinuous and its level sets are compact. Thus, it is immediate to check that $\Psi(K)=$ $\{g: I(g) \leqslant 1\}$.

Remark 3.3. From Remark 2.6, it follows that the asymptotic behaviour of the normalized random walk turns out to be the same as the sequence 


$$
D_{1 / \sqrt{n \ln \ln n}} \tilde{T}^{n},
$$

where $\tilde{T}^{n}=\tilde{X}_{n}$ and the process $\tilde{X}$ is the principal invariant diffusion on $G$, that is, the diffusion process whose generator has the form

$$
L=\frac{1}{2} \sum_{i=1}^{i_{1}} \chi_{i}^{2}
$$

Indeed, using the notation introduced in Remark 2.6, the drift and the diffusion coefficient of $\tilde{Y}_{t}^{n}=D_{1 / \sqrt{n \ln \ln n}} \tilde{X}_{n t}$ are given by

$$
\bar{b}_{n}(y)=\frac{1}{2 \ln \ln n} \sum_{i=1}^{i_{1}} \nabla_{y} \chi_{i}(y) \cdot \chi_{i}(y), \quad \bar{\sigma}_{n}(y)=\sigma(y)=\left[\chi_{1}(y) \ldots \chi_{i_{1}}(y) 0 \ldots 0\right],
$$

so that $\bar{b}_{n} \rightarrow 0$ as $n \rightarrow \infty$. Thus, the statement of Theorem 3.1 holds for the sequence $\left\{\tilde{Y}^{n}\right\}_{n}$ as well, so that $\left\{D_{1 / \sqrt{n \ln \ln n}} \tilde{T}^{n}\right\}_{n}$ and $\left\{D_{1 / \sqrt{n \ln \ln n}} T^{n}\right\}_{n}$ have the same set of limit points.

In the particular case of $G=\mathbb{R}^{d}$, where the length of the group is $l=1-$ that is, $i_{1}=d$ - and $T^{n}$ becomes the standard sum of $n$ i.i.d. centred random variables, such a property is well known, since here the principal invariant diffusion is given by a standard Brownian motion on $\mathbb{R}^{d}$. Thus, for general groups, the role of the Brownian motion is played by the principal invariant diffusion.

Finally, let us observe that a more general functional law can be stated, similar to that given in Baldi (1986). Indeed, in that paper a law of the iterated logarithm is proved whenever $\tilde{X}$ is (almost) any invariant diffusion on $G$, that is, its infinitesimal generator has the form

$$
L=\mathscr{H}_{0}+\sum_{i=1}^{r} \mathscr{Y}_{i}^{2}
$$

where, for $i=0, \ldots, r \leqslant d, \mathscr{Y}_{i}$ stands for the left-invariant vector field corresponding to (a fixed) $y_{i} \in \mathfrak{G}$ :

$$
\mathscr{Y}_{i} f(y)=\left.\frac{\mathrm{d}}{\mathrm{d} t} f\left(y \circ t y_{i}\right)\right|_{t=0} .
$$

The drift and the diffusion coefficients are given by

$$
\tilde{b}(y)=\mathscr{H}_{0}(y)+\sum_{i=1}^{r} \nabla_{y} \mathscr{Y}_{i}(y) \cdot \mathscr{H}_{i}(y), \quad \tilde{\sigma}(y)=\left[\mathscr{Y}_{1}(y) \ldots \mathscr{Y}_{r}(y) 0 \ldots 0\right] .
$$

Now, if $y_{0} \notin V_{1}$, then a law of the iterated logarithm follows. It is worth noticing that the constraint $y_{0} \notin V_{1}$ does not mean that $\tilde{X}_{t}$ is centred on $G$ (i.e. $\mathrm{E}\left[P_{1} \tilde{X}_{t}\right]=0$ ), but ensures that, as $n \rightarrow \infty, \mathrm{E}\left[P_{1} D_{1 / \sqrt{n \ln \ln n}} \tilde{X}_{t}\right] \rightarrow 0$, because this is equivalent to requiring that $\bar{b}_{n}$ converges as $n \rightarrow \infty$, where $\bar{b}_{n}$ is the drift of the diffusion $D_{1 / \sqrt{n \ln \ln n}} \tilde{X}_{n t}, t \in[0,1]$. Notice that such a fact is indeed what we need in the proof of Theorem 3.1.

Now, one could consider an analogous result for the case of ODEs: taking $\hat{b}=\tilde{b}$, or simply $\hat{b}=\mathscr{H}_{0}$, and $\hat{\sigma}=\tilde{\sigma}$, then the process $X$ which solves 


$$
\begin{aligned}
& \dot{X}_{t}=\hat{b}\left(X_{t}\right)+\hat{\sigma}\left(X_{t}\right) \dot{S}_{t}, \\
& X_{0}=0,
\end{aligned}
$$

satisfies a law of the iterated logarithm: if $y_{0} \notin V_{1}$, the sequence $\left\{D_{1 / \sqrt{n \ln \ln n}} X_{n}\right\}_{n}$ is a.s. relatively compact on $\mathscr{C}([0,1], G)$ and the set of its limit points is the set $K$ in Theorem 2.5 , in which

$$
b=0, \quad \sigma=\left[\mathscr{H}_{1} \ldots \mathscr{Y}_{r} 0 \ldots 0\right] .
$$

For the proof, it is sufficient to recall Remark 2.6 and Theorem 4.1 in Baldi (1986).

\section{An example: the Heisenberg group}

This section is devoted to an application of Theorem 3.2 to the simplest Lie group in the class of interest: the Heisenberg group.

Let $\mathbb{G}$ be the Lie algebra on $\mathbb{R}^{2 k+1}$, with $k \geqslant 1$, spanned by $\left\{e_{1}, \ldots, e_{2 k+1}\right\}$, with

$$
\left[e_{i}, e_{j}\right]= \begin{cases}e_{2 k+1} & \text { if } j=i+k, 1 \leqslant i \leqslant k \\ -e_{2 k+1} & \text { if } j=i-k, k+1 \leqslant i \leqslant 2 k \\ 0 & \text { otherwise. }\end{cases}
$$

Here $l=2, V_{1}$ is generated by $\left\{e_{1}, \ldots, e_{2 k}\right\}$ and $V_{2}$ by $\left\{e_{2 k+1}\right\}$. By the CampbellHausdorff formula, the left invariant vector fields associated with $e_{1}, \ldots, e_{2 k+1}$ can be written as

$$
\chi_{i}(y)= \begin{cases}\frac{\partial}{\partial y_{i}}-\frac{1}{2} y_{i+k} \frac{\partial}{\partial y_{2 k+1}} & \text { if } 1 \leqslant i \leqslant k \\ \frac{\partial}{\partial y_{i}}+\frac{1}{2} y_{i-k} \frac{\partial}{\partial y_{2 k+1}} & \text { if } k+1 \leqslant i \leqslant 2 k \\ \frac{\partial}{\partial y_{2 k+1}} & \text { if } i=2 k+1 .\end{cases}
$$

The Heisenberg group $\mathbb{H}_{k}$ on $\mathbb{R}^{2 k+1}$ is the Lie group having $\mathbb{G}$ as its Lie algebra. Again from the Campbell-Hausdorff formula, the product on $\mathbb{H}_{k}$ turns out to be

$$
g \circ \hat{g}=\left(g_{1}+\hat{g}_{1}, \ldots, g_{2 k}+\hat{g}_{2 k}, g_{2 k+1}+\hat{g}_{2 k+1}+\frac{1}{2} \sum_{i=1}^{k}\left(g_{i} \hat{g}_{i+k}-g_{i+k} \hat{g}_{i}\right)\right)
$$

and the endomorphism $D_{\alpha}$ becomes

$$
D_{\alpha} g=\left(\alpha g_{1}, \ldots, \alpha g_{2 k}, \alpha^{2} g_{2 k+1}\right) .
$$

Let $\left\{Z^{j}\right\}_{j}$ be a sequence of i.i.d. random variables taking values in $\mathbb{H}_{k}$. If $T^{n}=$ $Z^{1} \circ \ldots \circ Z^{n}$, it is easy to check that 


$$
\begin{aligned}
T_{j}^{n} & =\sum_{i=1}^{n} Z_{j}^{i}, \quad j=1, \ldots, 2 k, \\
T_{2 k+1}^{n} & =\sum_{i=1}^{n} Z_{2 k+1}^{i}+\frac{1}{2} \sum_{i=2}^{n} \sum_{j=1}^{i-1} \sum_{s=1}^{k}\left(Z_{s}^{j} Z_{s+k}^{i}-Z_{s+k}^{i} Z_{s}^{j}\right) .
\end{aligned}
$$

Theorem 3.2 allows a law of the iterated logarithm to be deduced for the random walk $\left\{T^{n}\right\}_{n}$ : the limit set of the sequence $\left\{D_{1 / \sqrt{n \ln \ln n}} T^{n}\right\}_{n}$ is given by

$$
K_{1}=\left\{g: g=x_{1}, x \text { such that } J(x) \leqslant 1\right\}=\{g: I(g) \leqslant 1\},
$$

where $J$ is as in (13), with

$$
b(y)=0, \quad \sigma_{i j}(y)= \begin{cases}1 & \text { if } i=j \leqslant 2 k, \\ -\frac{1}{2} y_{i+k} & \text { if } j=2 k+1,1 \leqslant i \leqslant k, \\ \frac{1}{2} y_{i-k} & \text { if } j=2 k+1, k+1 \leqslant i \leqslant 2 k, \\ 0 & \text { otherwise }\end{cases}
$$

and where

$$
I(g)=\inf _{x: x_{1}=g} J(g)
$$

The problem of computing such an infimum exactly is a non-trivial variational problem, because the matrix field $\sigma$ is degenerate in the sense that $\operatorname{det} \sigma \sigma^{\mathrm{T}}(y)=0$ for any $y$. Nevertheless, it has been solved through the associated Hamiltonian system in Gaveau (1977): if the covariance matrix is the identity, recalling that $P_{1} g=\left(g_{1}, \ldots, g_{2 k}\right)$ and $P_{2} g=g_{2 k+1}$,

$$
I(g)= \begin{cases}\frac{1}{2}\left|P_{1} g\right|^{2} & \text { if } P_{2} g=0, \\ 2 \pi\left|g_{2 k+1}\right| & \text { if } P_{1} g=0, \\ \frac{1}{2}\left|P_{1} g\right|^{2} \tau^{2}\left(\frac{4\left|g_{2 k+1}\right|}{\left|P_{1} g\right|^{2}}\right)\left(\sin \tau\left(\frac{4\left|g_{2 k+1}\right|}{\left|P_{1} g\right|^{2}}\right)\right)^{-2} & \text { otherwise, }\end{cases}
$$

where $\tau$ is the inverse function of

$$
\theta:\left[0, \pi\left[\rightarrow \left[0,+\infty\left[, \quad \theta(t)=\frac{2 t-\sin (2 t)}{2 \sin ^{2} t} .\right.\right.\right.\right.
$$

In the case of the Heisenberg group, the principal invariant diffusion on $\mathbb{H}_{k}$ is given by

$$
\tilde{X}_{t}=\left(B_{1}(t), \ldots, B_{2 k}(t), \frac{1}{2} \sum_{i=1}^{k} \int_{0}^{t}\left[B_{i}(s) \mathrm{d} B_{i+k}(s)-B_{i+k}(s) \mathrm{d} B_{i}(s)\right]\right),
$$

where $B=\left(B_{1}, \ldots, B_{2 k}\right)$ denotes a $2 k$-dimensional standard Brownian motion. Thus, as discussed in Remark 3.3, it follows that the asymptotic behaviour of $\left\{D_{1 \sqrt{n \ln \ln n}} T^{n}\right\}_{n}$ is the same as 


$$
\left(\frac{1}{\sqrt{n \ln \ln n}} B_{1}(n), \ldots, \frac{1}{\sqrt{n \ln \ln n}} B_{2 k}(n), \frac{1}{2 n \ln \ln n} \sum_{i=1}^{k} \int_{0}^{n}\left[B_{i}(s) \mathrm{d} B_{i+k}(s)-B_{i+k}(s) \mathrm{d} B_{i}(s)\right]\right) .
$$

Consider the case $k=1$. Concerning the third component, whose asymptotic behaviour has already been investigated by Crepel and Roynette (1977), the sequence defined by

$$
\frac{1}{n \ln \ln n}\left(\sum_{i=1}^{n} Z_{3}^{i}+\frac{1}{2} \sum_{i=2}^{n} \sum_{j=1}^{i-1}\left(Z_{1}^{j} Z_{2}^{i}-Z_{2}^{j} Z_{1}^{i}\right)\right)
$$

is obviously also a.s. relatively compact, and it immediately follows that the set of its limit points is $\left\{\hat{g} \in \mathbb{R} \mid I_{3}(\hat{g}) \leqslant 1\right\}$, where

$$
I_{2}(\hat{g})=\inf _{g \in \mathbb{H}_{3}: g_{3}=\hat{g}} I(g)=\inf _{\left(g_{1}, g_{2}\right) \in \mathbb{R}^{2}} I\left(g_{1}, g_{2}, \hat{g}\right) .
$$

Now, if $\hat{g}=0$ then obviously $I_{3}(\hat{g})=0$. Otherwise, notice that, if $P_{1} g \neq 0$, then

$$
I_{2}(g)=2\left|g_{3}\right| \cdot \frac{\left|P_{1} g\right|^{2}}{4\left|g_{3}\right|} \tau^{2}\left(\frac{4\left|g_{3}\right|}{\left|P_{1} g\right|^{2}}\right)\left(\sin \tau\left(\frac{4\left|g_{3}\right|}{\left|P_{1} g\right|^{2}}\right)\right)^{-2}
$$

so that

$$
\inf _{\left(g_{1}, g_{2}\right) \in \mathbb{R}^{2} \backslash\{0\}} I\left(g_{1}, g_{2}, \hat{g}\right)=2|\hat{g}| \cdot \inf _{\xi>0}\left(\frac{1}{\xi} \tau^{2}(\xi)(\sin \tau(\xi))^{-2}\right)=2|\hat{g}| \cdot \inf _{t \in(0, \pi)} \frac{t^{2}}{\theta(t) \sin ^{2} t}=\pi|\hat{g}| .
$$

Therefore,

$$
I_{3}(\hat{g})=\pi|\hat{g}|,
$$

so that the set of the limit points is $[-1 / \pi, 1 / \pi]$.

If the random variables $Z^{j}$ do not have the identity as their covariance matrix, a simple transformation easily enables the set of the limit points to be represented. Indeed, first of all, since by the strong law of the large numbers, $(n \ln \ln n)^{-1} \sum_{i=1}^{n} Z_{3}^{i} \rightarrow 0$ a.s. as $n \rightarrow \infty$, it is sufficient to study

$$
\frac{1}{2 n \ln \ln n} \sum_{i=2}^{n} \sum_{j=1}^{i-1}\left(Z_{1}^{j} Z_{2}^{i}-Z_{2}^{j} Z_{1}^{i}\right)
$$

Let $\Lambda$ denote the covariance matrix of the subvector $\left(Z_{1}^{i}, Z_{2}^{i}\right)$. Let $M$ be a $2 \times 2$ matrix such that $\Lambda=M M^{*}$, and $\left\{\tilde{Z}^{i}\right\}_{i}$ be a sequence of i.i.d. $\mathbb{R}^{2}$-valued random variables having the $2 \times 2$ identity as covariance matrix, such that $Z^{i}=M \tilde{Z}^{i}$. Then, it can easily be seen that

$$
\frac{1}{2 n \ln \ln n} \sum_{i=2}^{n} \sum_{j=1}^{i-1}\left(Z_{1}^{j} Z_{2}^{i}-Z_{2}^{j} Z_{1}^{i}\right)=\frac{\operatorname{det} M}{2 n \ln \ln n} \sum_{i=2}^{n} \sum_{j=1}^{i-1}\left(\tilde{Z}_{1}^{j} \tilde{Z}_{2}^{i}-\tilde{Z}_{2}^{j} \tilde{Z}_{1}^{i}\right) \text {. }
$$

Since the limit set of the sequence on the right-hand side is given by $[-1 / \pi, 1 / \pi]$, then the set of the limit points of $\left\{1 / 2 n \ln \ln n \sum_{k=2}^{n} \sum_{j=1}^{k-1}\left(Z_{1}^{j} Z_{2}^{k}-Z_{2}^{j} Z_{1}^{k}\right)\right\}_{n}$ is the closed interval 


$$
\left[-\frac{C}{\pi}, \frac{C}{\pi}\right]
$$

where $C=0$ if $\operatorname{det} M=0$, or otherwise

$$
\left.C=(\operatorname{det} M)^{-1}=(\operatorname{det} \Lambda)^{-1 / 2}=\left(\mathrm{E}\left[\left(Z_{1}^{1}\right)^{2}\right] \mathrm{E}\left[\left(Z_{2}^{1}\right)^{2}\right]-\mathrm{E}^{2}\left[Z_{1}^{1} \cdot Z_{2}^{1}\right]\right]\right)^{-1 / 2},
$$

a constant which has been determined in Crepel and Roynette (1977).

Therefore, when all the moments of $Z^{1}$ are finite or, more precisely, when its Laplace transform is finite in a neighbourhood of the origin, our result extends that proved by Crepel and Roynette, since they were able to determine the asymptotic behaviour of normalized random walks on $\mathbb{H}_{3}$ only componentwise.

Finally, let us observe that (33) gives the rate function associated with the projection on $V_{2}$ also in the case $k>1$. Therefore, at least when the covariance matrix is the identity, the closed interval $[-1 / \pi, 1 / \pi]$ provides the limit set of the projection on $V_{2}$ for the random walk on $\mathbb{H}_{k}$, which is usually called the 'central component'.

\section{References}

Baldi, P. (1986) Large deviation and functional iterated logarithm law for diffusion processes. Probab. Theory Related Fields, 71, 435-453.

Baldi, P. and Caramellino, L. (1999) Large and moderate deviations for random walks on nilpotent groups. J. Theoret. Probab., 12, 779-809.

Caramellino, L., Climescu-Haulica, A. and Pacchiarotti, B. (1999) Diffusion approximations for random walks on nilpotent Lie groups. Statist. Probab. Lett., 41, 363-377.

Crepel, P. and Roynette, B. (1977) Une loi du logarithme itéré pour le groupe de Heisenberg. Z. Wahrscheinlichkeitstheorie Verw. Geb., 39, 217-229.

Gaveau, B. (1977) Principe de moindre action, propagation de la chaleur et estimées sous-elliptiques sur les groupes nilpotents. Acta. Math., 139, 95-153.

Mogul'skii, A.A. (1976) Large deviations for trajectories of multidimensional random walks. Theory Probab. Appl., 21, 309-323.

Neuenschwander, D. (1996) Probabilities on the Heisenberg Group. Limit Theorems and Brownian Motion, Lecture Notes in Math. 1630. Berlin: Springer-Verlag.

Strassen, V. (1964) An invariance principle for the law of the iterated logarithm. Z. Wahrscheinlichkeitstheorie Verw. Geb., 3, 211-226.

Received July 1999 and revised March 2001 